\title{
Use of an accident and emergency department by hospital staff
}

\author{
C J Mann
}

\begin{abstract}
Objective-To assess the number of attendances by hospital staff at an accident and emergency (A\&E) department, and reasons for their attendance.

Methods-A\&E attendances by hospital staff were studied for a 12 month period. Comparison was made with attendances by non-hospital staff in full or part time employment. Differences between the observed and expected numbers of attendances were analysed using $\chi^{2}$ analysis.

Results—560 staff attendances were recorded out of 78103 total attendances. There was an observed excess number of attendances by nursing staff for work related incidents when compared to the control group $(P=0.01)$. However, there were fewer attendances by nursing staff for non-work related incidents $(P=0.01)$. Staff other than doctors and nurses attended the A\&E department more frequently than the control group for nonworkrelated incidents $(P=0.01)$, but their attendance for incidents occurring at work were the same as the control group (P = NS).

Conclusions-The increased use of A\&E resources by staff other than doctors and nurses may be inappropriate and further research into their reasons for attendance is warranted.

(F Accid Emerg Med 1996;13:409-411)
\end{abstract}

Key terms: accident and emergency departments; hospital staff attendances

Whenever clinical staff repeatedly attend an accident and emergency (A\&E) department for non-work-related incidents the reasons for the attendance should be examined and appropriate action taken.

The use of an A\&E department by hospital staff for occupational health reasons is inevitable and should be planned for. A system to protect confidentiality must be in place and the records kept securely to prevent unauthorised access.

The general population attend A\&E departments principally for the diagnosis and treatment of acute illness and injury. ${ }^{1}$ The staff of a hospital form a subset of the patients attending an $A \& E$ department and their perception of the role of the $A \& E$ department may be quite different from that of the general public. Several studies have shown that a proportion of attendances by the general public may be described as "inappropriate" or "primary care attendances", ${ }^{3}$ and estimates of the number of such patients vary from $6.7 \%^{3}$ to $65 \%$. $^{4}$ To our knowledge there are no reports on the use of A\&E departments by hospital staff.

Recently the media have highlighted an occasion when such attendances should have been brought to the attention of senior medical staff. ${ }^{5}$ The official inquiry into the Beverly Allitt case suggested that recurrent attendances by an individual member of staff should have been investigated for the benefit of the individual and others. ${ }^{6}$

In the present study we assessed the use of the A\&E department by hospital staff and compared it with the use made by the general public. During the study year the A\&E department of Queen Alexandra Hospital served a population of $543000 .^{7}$ Of these 251000 were in full or part time employment ${ }^{8} ; 3425$ were employed by the Portsmouth Health Trust and of those $318(9 \%)$ were doctors, $1530(44 \%)$ were nurses, and 1577 (47\%) were employed in other activities.

\section{Methods}

The A\&E records for all staff working for the Trust attending the A\&E department over a 12 month period in 1993 to 1994 were examined retrospectively. They were compared with an equivalent number of randomly selected $A \& E$ records of the general public aged between 16 and 65 who declared themselves to be in full or part time employment.

Hospital staff attendances are routinely recorded at time of presentation. These were reviewed to define attendance by three different groups; doctors, nurses and other staff.

Admission rates, follow up rates, referrals to general practitioner, and discharge were measured as an indicator of casemix..$^{9}$

The findings were evaluated using $\chi^{2}$ analysis.

\section{Results}

OVERALL ATTENDANCES

There were 78103 new attendances at $\mathrm{A} \& \mathrm{E}$ during the study period $(14.6 \%$ of population served); 28650 patients declared themselves to be in full or part time employment $(11.4 \%$ of the working population). A\&E attendances by Trust employees numbered $560(16.35 \%$ of the work force) $(P=0.01)$.

During the study period, 696 patients presented to the occupational health service with either illness or injury. Thus A\&E accounted for $45 \%$ of all staff attendances to $\mathrm{A} \& \mathrm{E}$ and the occupational health service combined. 


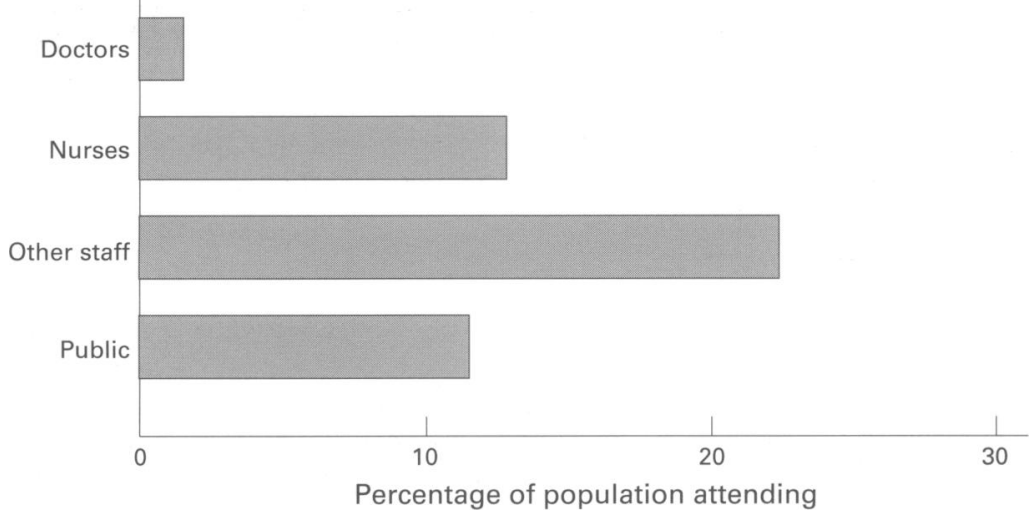

Figure 1 Comparison of attendances by Trust staff and the general public.

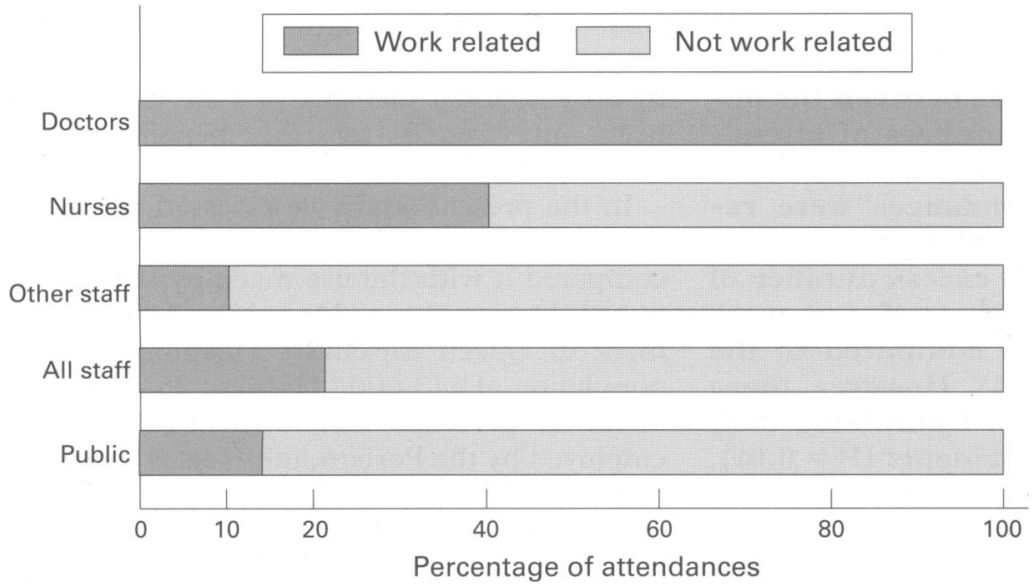

Figure 2 Incidents and their relations to employment.

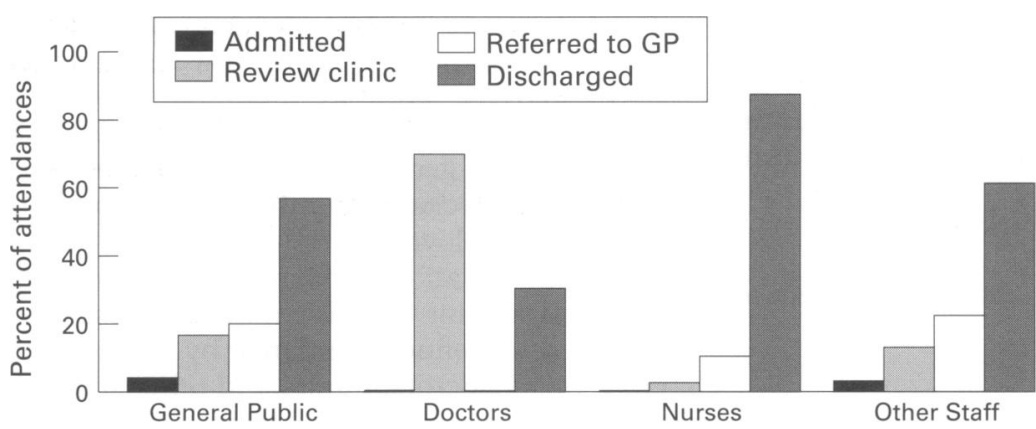

Figure 3 Outcome of work related incidents.

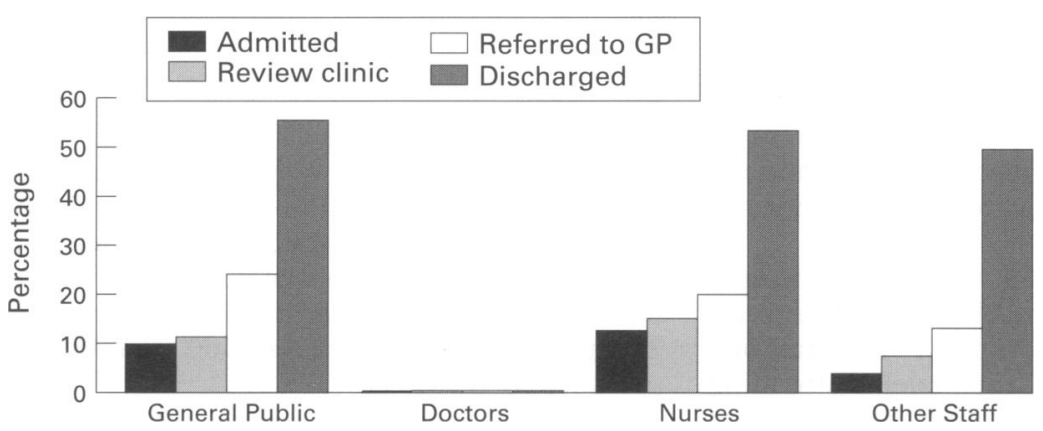

Figure 4 Outcome of non-work-related incidents.

Doctors made five attendances $(0.9 \%)$ to A\&E, nurses 199 (35.5\%), and other staff made $356(63 \%)$ attendances.
Figure 1 shows that staff attendances in excess of those predicted were due to nursing $(P=0.05)$ and other staff $(P=0.01)$. Doctors attended less frequently than all other groups.

WORK RELATED INCIDENTS

Work related incidents accounted for $10 \%$ of the attendances of the general public in employment and $21.4 \%$ of those by hospital staff $(P=0.01)$

Although doctors only attended as a result of work related incidents, the total number of attendances (5) was too small to allow statistical analysis. For nursing staff, work related incidents accounted for $40 \%$ of attendances, four times the proportion recorded for the general public $(P=0.01)$.

The attendance rate for work related incidents in other staff was identical to that of the general population (10\%) (fig 2 ).

NON-WORK-RELATED INCIDENTS

Nursing and medical staff attended less frequently than the public for non-workrelated incidents $(P=0.01)$. There was an observed excess of total attendances for non-work-related incidents in other hospital staff $(P=0.01)$ (fig 1$)$

OUTCOME OF ATTENDANCES

The seriousness of each incident was defined by its outcome (admission, clinic review, referral to GP, or discharge).

Work related incidents

Doctors' attendances were too few to allow valid comparisons with the other groups studied. Nursing staff attendances seldom required further follow up when compared to the general population $(P=0.05)$, implying that their injuries were relatively minor or that they were better able to cope. Outcomes for other staff following work related incidents were not statistically different from the general public (fig 3).

\section{Non-work-related incidents}

Figure 4 shows the outcomes of non-workrelated incidents. There were no attendances by doctors. The attendance pattern of nursing staff was not significantly different from that of the general public. Other staff were admitted, reviewed in clinic, or referred to their general practitioner less often than the general public $(\mathrm{P}=0.05)$.

\section{Discussion}

Our results. show that a significantly greater percentage of hospital staff attended the A\&E department of their own hospital than would be expected by comparison with the general population. This was true for all groups of hospital staff except doctors. However, if work related incidents are excluded, doctors and nurses attended less frequently than expected. Other staff attended more often than the general population for incidents unrelated to work. 
WORK RELATED INCIDENTS

Work related incidents that cause nursing staff to attend $A \& E$ are often minor in nature compared to other staff and the general public, or are a consequence of their employment, for example, needle-stick injuries. The main reason for attending is to enable official documentation of all work related injuries, essential in the event of consequential sick leave or compensation claims.

The high attendance rate also reflects the disparity between a 24 hour / seven day a week nursing service and an 8 hour a day / five day a week occupational health service. This compels $A \& E$ departments to fulfil part of the role of an occupational health service.

\section{NON-WORK-RELATED INCIDENTS}

The high attendance rate to $A \& E$ by other staff is a cause for concern. It is possible that familiarity with the hospital environs and awareness of a 24 hour service cause them to regard the $\mathrm{A} \& \mathrm{E}$ department as a more convenient health care resource than their general practitioners. This has implications for them and the department. Most A\&E departments are not designed to provide a general practice service, and patients attending the department for advice and treatment of problems more usually dealt with by general practitioners may be disadvantaged.

Reasons for low attendance rates to $A \& E$ by doctors may include greater insight of illness and injury, embarrassment at being treated by their peers and juniors, and the ability (albeit ill advised) to self diagnose and treat. Nursing staff share many of these characteristics. Furthermore, both groups may participate in "corridor consultations" where undocumented advice is given. Because of the nature of these consultations it is not possible to quantify how often this occurs.

Because attendance to an $\mathrm{A} \& \mathrm{E}$ department by a doctor or nurse for an incident unrelated to work is uncommon, recurrent attendances by an individual doctor or nurse should trigger a review by a senior clinician. This would not compromise confidentiality but would allow both the clinician and the attender to examine the reasons for these attendances.

\section{CONCLUSION}

The increased use of $A \& E$ resources by staff other than doctors and nurses appears to be inappropriate and further research into their reasons for attendance is warranted. Whenever clinical staff repeatedly attend an A\&E department for non-work-related incidents the reasons for the attendance should be examined and appropriately acted upon. The use of an A\&E department by hospital staff for occupational health reasons is inevitable and should be planned for. A\&E staff require a working knowledge of the occupational health service and will need some training to prevent hospital staff presenting out of hours receiving a lesser standard of care than those presenting to occupational health within hours.

A system to protect confidentiality must be in place and the records kept securely to prevent unauthorised access.

1 Driscoll PA, Vincent CA. The use of the accident and emergency department. Arch Emerg Med 1987;4:77-82.

2 Foroughi D, Chadwick L. Accident and emergency abusers. Practitioner 1988;233:657-9.

3 British Association for Accident and Emergency Medicine. Relationship between accident and emergency medicine and primary care. Report of the Association, London, August 1994.

4 Crombie DL. A casualty survey. $\mathcal{F}$ R Coll Gen Pract 1959;2: 346-56.

5 The Guardian, 18 May, 1993.

6 Report of the Sir Cecil Clothier enquiry. London: HMSO, 1993.

7 The health services yearbook. London: Institute of Health Services Management, 1994.

8 Community health atlas. Portsmouth: Portsmouth and SE Hampshire Health Commission, 1993

9 Anthony P, Blayden B, Bray P. Casemix measures in acciden and emergency medicine: an introductory review. National Casemix Office, Romsey Rd, Winchester, 1995. 